\title{
Modelagem Matemática para a Simulação Computacional de uma Solução de Integração no Contexto da Administração Pública de Ijuí-RS
}

\author{
Alexsandro Queiroz Lencina ${ }^{1}$ \\ Instituto Federal de Educação, Ciência e Tecnologia Farroupilha, IFFar, São Borja, RS \\ Rafael Zancan Frantz ${ }^{2}$ \\ Departamento de Ciências Exatas e Engenharias, Modelagem Matemática, UNIJUÍ, Ijuí, RS \\ Fabricia Roos-Frantz ${ }^{3}$ \\ Departamento de Ciências Exatas e Engenharias, Modelagem Matemática, UNIJUÍ, Ijuí, RS \\ Sandro Sawicki ${ }^{4}$ \\ Departamento de Ciências Exatas e Engenharias, Modelagem Matemática, UNIJUí, Ijuí, RS
}

Resumo. A grande maioria das empresas possui um ecossistema de software composto por um conjunto de aplicações, para auxiliar na tomada de decisões ou na melhoria dos seus processos de negócio. O campo de estudo Integração de Aplicações Empresarias oferece ferramentas e metodologias para criar soluções de integração que possibilitam que as aplicações do ecossistema, geralmente heterogêneas, possam trabalhar de maneira conjunta e sincronizada. Este trabalho utiliza como caso de estudo uma solução de integração projetada para a geração dos boletos do Imposto Predial e Territorial Urbano (IPTU) e do Imposto sobre Serviços de Qualquer Natureza (ISSQN) da Prefeitura no município de Ijuí RS. Esta solução, projetada com uma linguagem de modelagem de alto nível de abstração, é caracterizada como um sistema de eventos discretos. Existem vários paradigmas para a modelagem de sistemas de eventos discretos, dentre eles aqueles baseados em modelos probabilísticos. Neste trabalho, utiliza-se uma abordagem baseada em simulação com redes de Petri Estocásticas para a identificação de gargalos de desempenho em soluções de integração, caracterizando, assim, uma solução como um processo probabilístico. Para a redução desses gargalos utilizou-se as redes de Petri Estocásticas Generalizadas. A simulação de uma solução de integração, a partir do seu modelo conceitual, possibilita a análise do seu comportamento ainda na fase de projeto, reduzindo custos, riscos e tempo no desenvolvimento da solução. Os resultados da simulação mostram que os gargalos de desempenho podem ser identificados ainda na fase de projeto e que é possível reduzí-los dedicando recursos computacionais às tarefas mais críticas do modelo.

Palavras-chave. Integração de Aplicações Empresariais, Redes de Petri, Simulação, Gargalos de Desempenho.

\footnotetext{
${ }^{1}$ alexsandro.queiroz@iffarroupilha.edu.br

${ }^{2}$ rzfrantz@unijui.edu.br

${ }^{3}$ frfrantz@unijui.edu.br

${ }^{4}$ sawicki@unijui.edu.br
} 


\section{Introdução}

A grande maioria das organizações possui um conjunto de aplicações de software que dão suporte a suas atividades de negócio, o qual é denominado ecossistema de software. Normalmente essas aplicações são heterogêneas, concebidas sem levar em conta sua possível integração.

Na área de Engenharia de Software, o campo de estudos conhecido como Integração de Aplicações Empresariais [4] busca proporcionar metodologias, técnicas e ferramentas para a concepção e a implementação de soluções de integração. A finalidade dessa integração é orquestrar um conjunto de aplicações para mantê-las sincronizadas ou proporcionar novas funcionalidades que possam ser construídas a partir das já existentes.

A necessidade de integração fomentou o surgimento de várias tecnologias de integração de aplicações, com isso a necessidade de simulações, testes para a verificação da correta implementação dessas aplicações.

O objetivo deste trabalho é analisar o comportamento de uma solução de integração, proposta por [5], para verificar a possível identificação de gargalos de desempenho e dessa forma propor uma solução para a sua redução.

Na identificação dos possíveis gargalos utilizou-se as redes de Petri Estocásticas - Stochastic Petri Nets (SPN), tendo em vista que uma solução de integração pode ser definida como um sistema de eventos discretos e a execução dos seus disparos acontecem aleatoriamente. As redes de Petri (RdP) são ferramentas matemáticas que oferecem um ambiente para modelagem, análise e simulação desse tipo de sistema [6]. Após a análise das SPN, será possível identificar os possíveis gargalos de desempennho podendo assim dedicar recursos computacionais para os pontos mais críticos do sistema e tentar saná-los com auxílio das redes de Petri Estocásticas Generalizadas - Generalized Stochastic Petri Nets (GSPN).

As GSPN são um tipo de rede que permite a utilização de transições imediatas, as quais possuem prioridade sobre as demais transições. Diante disso opta-se pela rede tendo em vista uma possível redução para esses gargalos. Para a simulação utilizou-se a ferramenta Plataform Independent Petri Nets 2 - PIPE2.

Com o desenvolvimento deste tabalho será possível fazer a representação de um modelo conceitual de solução de integração em um modelo matemático, possibitando assim identificar, reduzir os possíveis gargalos de desempenho de um sistema, ainda em sua fase de projeto.

\section{Modelagem da Solução de Integração}

Para a modelagem do modelo conceitual, utilizou-se as RdP, pela relação análoga entre seus componentes, conforme a Tabela 1 e pela semelhança do modelo de execução. Os tokens das RdP são análogos às mensagens da solução de integração e os lugares são equivalentes aos slots, que atuam como buffers. Mesmo havendo uma equivalência entre componentes, cada tarefa do modelo conceitual tem uma semântica implementada que pode ser traduzida, que não foi feita neste trabalho. Foi criado um modelo de simulação que abstrai detalhes da semântica das tarefas. 
Tabela 1: Semelhança entre elementos DSL e RdP. [1

\begin{tabular}{|cc|cc|}
\hline \multicolumn{2}{|c|}{ Guaraná DSL } & \multicolumn{2}{|c|}{ Redes de Petri } \\
\hline$\square$ & Tarefa & & Transição \\
\hline$\ldots . \cdots$ & Slot & 0 & Lugar \\
\hline$\square$ & Mensagem & $\bullet$ & Token \\
\hline
\end{tabular}

Uma rede de Petri $R d P=\left(P, T, A, M_{o}\right)$ são grafos bipartidos compostos por dois elementos: um passivo denominado lugar e um ativo denominado transição. Representase os lugares $(P)$, transições $(T)$, arcos $(A)$ e uma marcação ou token inicial $M_{o}$, conforme Equação 1. Sua estrutura é formalmente especificada da seguinte maneira:

$$
R d P=\left(P, T, A, M_{o}\right),
$$

onde:

$$
\begin{aligned}
& P=\left\{p_{1}, p_{2}, p_{3} \ldots p_{n}\right\} \\
& T=\left\{t_{1}, t_{2}, t_{3} \ldots t_{n}\right\}, I \subseteq(P x T), \\
& O \subseteq(T x P), A=(I \cup O), \\
& M_{o}=\left\{M_{o}\left(p_{1}\right), M_{o}\left(p_{2}\right) \ldots M_{o}\left(p_{n}\right)\right\}
\end{aligned}
$$

Roos-Frantz [1], propõe a analogia entre a troca de estados em uma solução modelada com Guaraná e uma troca de estados em uma RdP, onde os lugares representam uma condição, um recurso e são representados graficamente por círculos e as transições representam um evento (ação) realizado pelo sistema são representados por um traço ou uma barra como é apresentado na Figura 1.

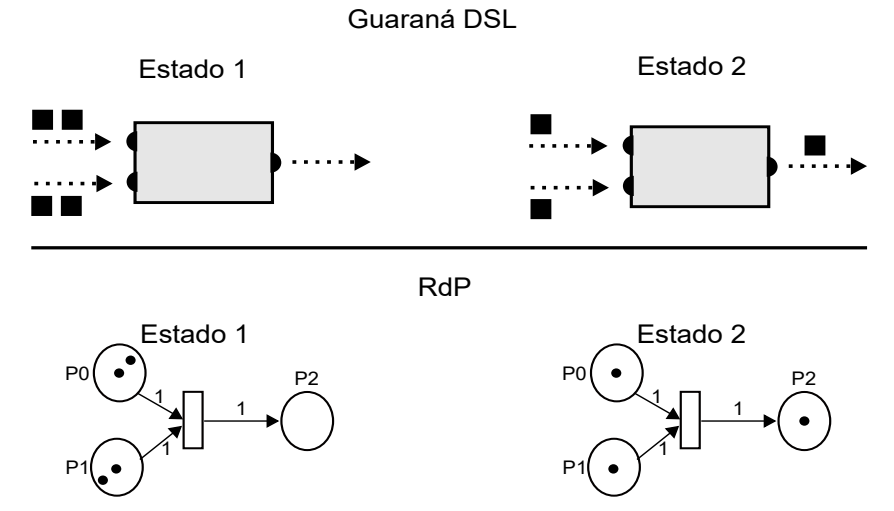

Figura 1: Semelhança entre estados DSL e RdP. [1]

A Figura 2 representa o modelo de simulação da solução de integração do estudo de caso, apresentado na Figura 3. A rede foi construída seguindo a tradução das tarefas em 
Guaraná DSL para as respectivas RdP. Estas redes estavam ligadas para cada estado na mesma forma das interligações das tarefas representadas na Figura 1.

$\mathrm{Na} \mathrm{RdP}$ resultante, as transições foram traduzidas de acordo com suas tarefas correspondentes e os lugares de acordo com seus respectivos slots, observando a organização e ligação das tarefas, a disposição das portas e a direção do fluxo de mensagens.

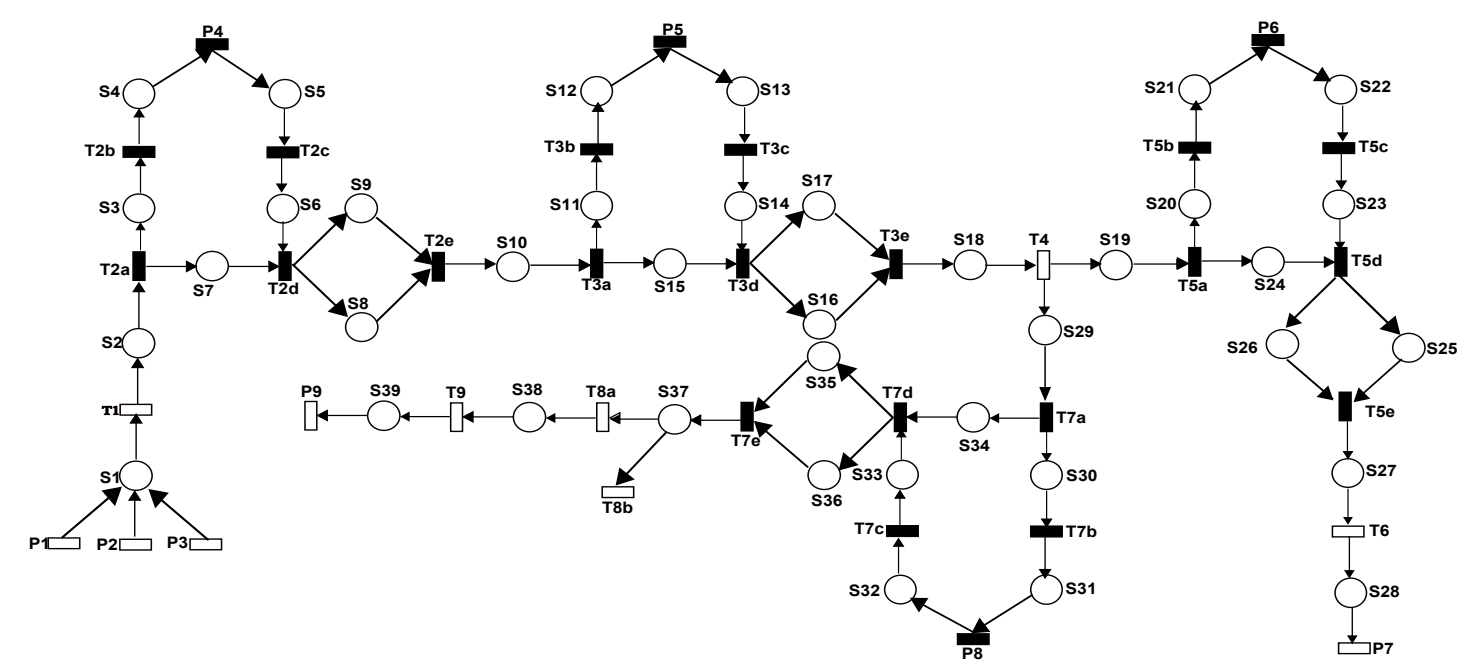

Figura 2: Modelo de Simulação.

\section{Experimento, Simulação e Análise dos Resultados}

A Figura 3 apresenta a solução de integração modelada em Guaraná DSL, uma linguagem de modelagem que permite modelar um processo de integração de aplicações em um alto nível de abstração [2]. Esta solução de integração foi projetada para um problema de integração no domínio da administração pública, identificado por Kuhne [5]. A partir de uma consulta com os profissionais do setor de informática da prefeitura municipal de Ijuí, RS, Brasil, identificou-se o ecossistema de software que dá suporte a geração de boletos do Imposto Predial e Territorial Urbano (IPTU) e do Imposto sobre Serviços de Qualquer Natureza (ISSQN). Ambos são impostos municipais de periodicidade anual e são calculados com base em informações contabilizadas pela Secretaria Municipal da Fazenda e seu ecossistema de software.

Para executar as simulações utilizou-se a ferramenta computacional PIPE2 [7], utilizouse em torno de 25 repetições, conforme sugerido em [3]. O resultado da simulação mostra a média de tokens acumulados em cada lugar da rede, com intervalo de confiança de $95 \%$, sem considerar o fator tempo.

Para a simulação, foi atribuído aleatoriamente 300 contribuintes, o que equivale a uma carga de entrada de 300 tokens, sendo um token para cada contribuinte. Para cada token no lugar de entrada (P1, P2 ou P3), serão necessários 33 disparos para que se gere um token nos lugares de saída [1]. Dessa forma, tomando como entrada 300 tokens, são necessários 


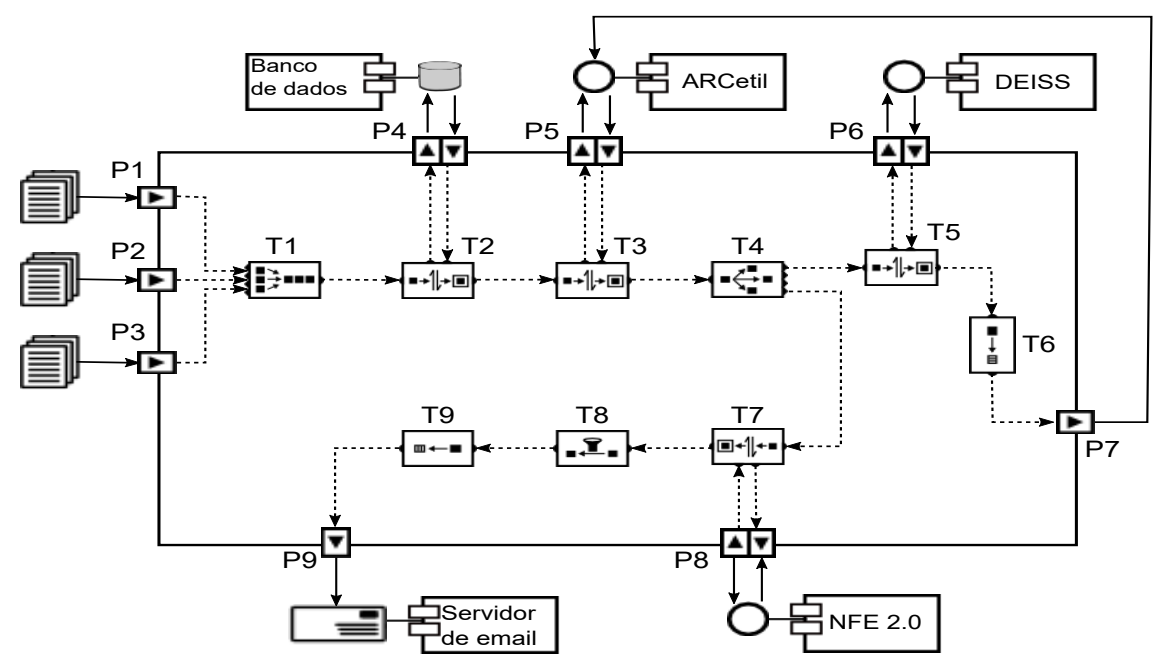

Figura 3: Solução de Integração. [5]

no mínimo 9900 disparos para simular o cenário proposto.

A primeira etapa na construção do modelo de simulação baseado em GSPN é a definição de quais as transições devem ser temporizadas e quais devem ser imediatas. Para isso, foi necessário identificar quais transições no modelo de simulação baseado em SPN geram gargalos de desempenho. Com a simulação da SPN verificou-se um acúmulo de mensagens nos slots S7, S15, S24 e S34. Isso se deve aos lugares que representam estes slots são lugares de entrada para as transições T2d, T3d, T5d e T7d, as quais só estarão habilitadas quando os lugares S6, S14, S23 e S33 possuírem tokens a serem processados.

Foram incluídos tarefas imediatas nas transições que faziam todo o processo de mensagem até chegarem nas transições que indicam gargalos, conforme a Figura 2, mais especificamente nas tarefas pertencentes a tarefa Enquirer do modelo conceitual, que é chamada de tarefa composta e sua composição é representada por outras quatro tarefas que são replicador, correlacionador, enriquecedor de conteúdo e transformador, conforme Figura 4.

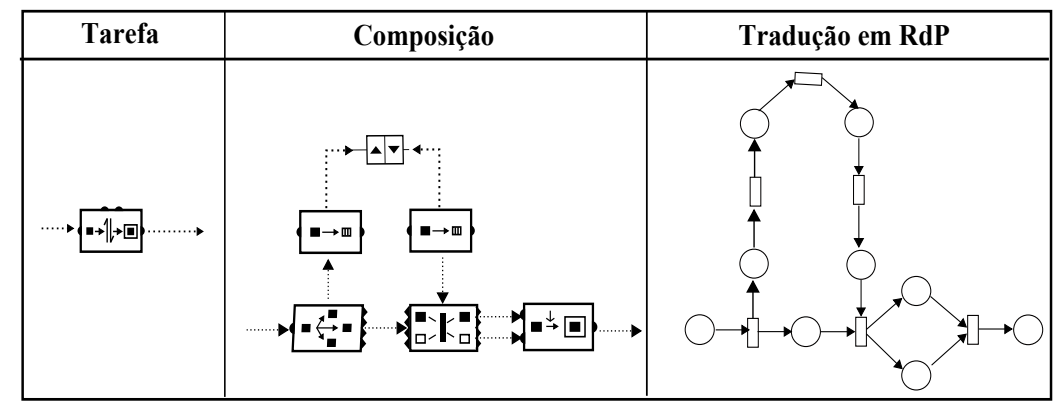

Figura 4: Tarefa Enquirer.

A simulação foi realizada para verificar se o gargalo diminuiria com o uso das transições 
imediatas, uma vez que os disparos destas transições tem prioridade e a sua ordem de execução deve ser definida, ou seja, não acontecem aleatoriamente, como ficaram mais de uma transição imediata a ordem foi definida pelo sentido do fluxo inicial de mensagens. Após a identificação das transições e a simulação observou-se uma distribuição proporcional/linear das mensagens no sistema, configurando assim a redução ou eliminação de gargalos de desempenho.

A identificação das transições que produzem gargalos e a atribuição de prioridade para estas transições, permitiu simular o comportamento da solução em caso de poder utilizar no sistema real mais recursos computacionais.

\section{Verificação do Modelo}

Para a verificação do modelo foi aplicada a proposta feita por Sargent [8] onde descrevese o fluxo de mensagens na perspectiva de verificar se o modelo está implementado corretamente, utilizou-se os dados iniciais sendo uma entrada de 9900 disparos. O modelo de simulação foi avaliado em setores e colocados contadores de disparos, a Figura 2, representa as GSPN. Este experimento foi executado uma vez, e sua intenção é descrever o fluxo de mensagem, com intuito de verificar se o modelo está implementado corretamente na ferramenta. Descreve-se abaixo por $\mathrm{n}(\mathrm{x})$ a quantidade de mensagens em todo o sistema e as referidas igualdades para atingir o objetivo da técnica:

$$
\begin{aligned}
& \mathrm{n}(\mathrm{P} 1, \mathrm{P} 2, \mathrm{P} 3, \mathrm{P} 4)=\mathrm{n}(\mathrm{S} 1 \ldots \mathrm{S} 10) \mathrm{U} \text { n(T1...T3a) } \\
& \mathrm{n}(\mathrm{T} 5 \mathrm{a})=\mathrm{n}(\mathrm{T} 3 \mathrm{a} \ldots \mathrm{T} 4) \mathrm{U} \text { n(S11...S19) U n(P5) } \\
& \mathrm{n}(\mathrm{P} 7)=\mathrm{n}(\mathrm{T} 5 \mathrm{a} \ldots \mathrm{T} 6) \mathrm{U} \text { n(S20..S28) U n(P6) } \\
& \mathrm{n}(\mathrm{P} 9)=\mathrm{n}(\mathrm{T} 4, \text { T7a...T9) U n(S29...S39) U n(P8) }
\end{aligned}
$$

Analisando os resultados, verifica-se que todos os setores satisfazem a premissa de partida da técnica Entrada = Filas + Saída. A verificação parte do número de disparos da transição que antecipa (entrada) o setor com valor de disparos dispostos nos marcadores de disparos e é igualada com o valor da transição de saída do setor (ou do modelo) somada as filas formadas no setor, havendo igualdade de valores o setor está verificado e não apresenta erros. Assim conclui-se que de acordo com a técnica de Sargent [8] que os modelos de simulação foram implementados de forma correta e no aspecto número de mensagens e suas filas não apresentaram erros em nenhum setor.

Portanto ao comparar o número de mensagens filtradas esperado com o obtido no experimento, pode-se constatar que seus valores são muito próximos, tratando-se de processos estocásticos, o que leva a concluir que há uma equivalência significativa nos eventos esperados no modelo conceitual e no modelo de simulação.

\section{Conclusões}

Este trabalho apresentou a modelagem e simulação de uma de solução de integração, utilizando redes Petri. O modelo conceitual da solução, projetado inicialmente com a 
tecnologia Guaraná, foi traduzido para um modelo de simulação em SPN e então analisado, para verificar a presença de gargalos de desempenho e a possível redução dos mesmos.

Com a simulação, identificou-se os possíveis gargalos de desempenho, ou seja, os locais onde ocorrem o maior acúmulo de mensagens no sistema, tudo isso na fase de projeto da solução de integração. Então, buscou-se com as GSPN reduzir os possíveis gargalos e as simulações foram submetidas a técnicas de verificação.

Portanto, um modelo conceitual de solução de integração pode ser representado por um modelo matemático e é possível identificar, reduzir ou eliminar os possíveis gargalos de desempenho de um sistema, ainda em sua fase de projeto.

Para trabalhos futuros, a proposta será aumentar a carga inicial e aproximar para a carga real de contribuintes, uma vez que para a simulação neste trabalho utilizamos uma carga para fins de estudos acadêmicos e de comprovação da proposta.

\section{Referências}

[1] F. R. Frantz, M. Binelo, R. Z. Frantz, S. Sawicki and V. B. Fernandes. Using Petri Nets to Enable the Simulation of Application Integration Solutions Conceptual Models, Proceedings of the 17th International Conference on Enterprise Information Systems - Volume 1: ICEIS, p. 87-96, 2015. DOI: 10.5220/0005351900870096.

[2] R. Z. Frantz, A. M. R. Quintero and R. Corchuelo. A domain-specific language to design enterprise application integration solutions, International Journal of Cooperative Information Systems, v.20, n.2, p.143-176, 2011. DOI: 10.1142/S0218843011002225.

[3] C. M. Grinstead and J. L. Snell. Introduction to probability. American Mathematical Soc., 2012.

[4] G. Hohpe and B. Woolf. Enterprise Integration Patterns: Designing, building, and deploying messaging solutions. Addison-Wesley Professional, 2004.

[5] I. E. M. Kuhne. Linguagem Guaraná DSL no Contexto da Administração Pública Municipal - Um Caso de Estudo. Trabalho de Conclusão de Curso, Universidade Regional do Noroeste do Estado Rio Grande do Sul, Ijuí, 2017.

[6] M. K. Molloy. Performance analysis using stochastic Petri nets. IEEE Transactions on computers, v. 31, n. 9, p. 913-917, 1982. DOI 10.1109/TC.1982.1676110.

[7] PIPE2: Platform Independent Petri Net Editor. URL: http://pipe2.sourceforge.net/.

[8] R. G. Sargent. Verification and validation of Simulation Models. Simulation Conference (WSC), Proceedings of the 2009 Winter. IEEE, p. 162-176, 2009. DOI: 10.1109/WSC.2007.4419595. 